\title{
A THEOREM ON THE COHOMOLOGY RING OF NILPOTENT GROUPS
}

\author{
TZEE-NAN KUO ${ }^{1}$
}

Let $G$ be a torsion free, finitely generated nilpotent group, and $H$ be a subgroup of finite index of $G$. Mal'cev proved in [4] that $G$ can be embedded in a connected, simply connected, nilpotent rational Lie group (5), such that $G$ and $H$ are discrete subgroups of $(B)$ and the coset spaces (3) $/ G$ and $\$ S / H$ are compact. Nomizu showed in [5] that if $L$ is the associated Lie algebra of $\$$, then $H^{n}\left(L, Q^{+}\right) \cong H^{n}\left(\$ / G, Q^{+}\right)$ where $Q^{+}$is the additive group of rational numbers. Let $H^{n}\left(G, Q^{+}\right)$ denote the rational cohomology group of the abstract group $G$. It follows from Lie group theory that $H^{n}\left(\$ / G, Q^{+}\right) \cong H^{n}\left(G, Q^{+}\right)$, hence $H^{n}\left(L, Q^{+}\right) \cong H^{n}\left(G, Q^{+}\right)$, and for the same reason we have $H^{n}\left(L, Q^{+}\right)$ $\cong H^{n}\left(H, Q^{+}\right)$, whence $H^{n}\left(G, Q^{+}\right) \cong H^{n}\left(H, Q^{+}\right)$.

In this paper we shall, however, indicate a purely algebraic approach to this theorem in the following strengthened form:

Theorem 1. If $G$ is a finitely generated nilpotent group and $H$ is a subgroup of finite index and $M$ is a trivial G-module which is torsion free and divisible, then $H^{n}(G, M) \cong H^{n}(H, M)$ via the restriction map for all $n \geqq 0$, and if in addition $M$ is a ring, then the restriction map is a ring isomorphism between $H^{*}(G, M)$ and $H^{*}(H, M)$.

Proof. Let

$$
\{e\}=A_{0} \subset A_{1} \subset \cdots \subset A_{n}=G
$$

be a central series of $G$. Since the factor groups $A_{i} / A_{i-1}$ of series (1) are finitely generated and abelian, it has a refinement

$$
\{e\}=B_{0} \subset B_{1} \subset \cdots \subset B_{m}=G
$$

with cyclic factor groups $B_{j} / B_{j-1}$ for all $j$. Let $k(G)$ be the number of infinite cyclic factor groups in (2). A finitely generated nilpotent group is an $S$-group in the sense of [1]. According to a result of Hirsch [1, Theorem 1.42, p. 58] $k(G)$ is independent of the choice of the central series, and if $N$ is a normal subgroup of $G$, then $k(G)=k(G / N)$

Received by the editors June 24, 1966.

1 This research was partially supported by National Science Foundation Grant NSF GP-4034. The contents of this paper formed a minor part of the doctoral dissertation of the author at the University of Chicago under the guidance of Professor J. L. Alperin. 
$+k(N)$ in as much as the series $G \triangleright N \triangleright\{e\}$ and the series (2) have common refinement.

It is well known [3, Vol. I, p. 83] that a subgroup $H$ of $G$ of finite index contains a normal subgroup $K$ of finite index in $G$. Since

$$
H^{n}(G, M) \stackrel{\text { res }}{\cong} B^{n}(K, M) \quad \text { and } \quad H^{n}(H, M) \stackrel{\text { res }}{\cong} H^{n}(K, M)
$$

would imply that

$$
H^{n}(G, M) \stackrel{\text { res }}{\cong} H^{n}(H, M),
$$

it suffices to show Theorem 1 under the assumption that $H$ is normal in $G$.

We shall prove our theorem by induction on $k(G)$. If $k(G)=0$, then $G$ is a finite group. By the Universal Coefficient Theorem we have the following exact sequence

$$
0 \rightarrow \operatorname{Ext}^{1}\left(H_{n-1}(G, Z), M\right) \rightarrow H^{n}(G, M) \rightarrow \operatorname{Hom}\left(H_{n}(G, Z), M\right) \rightarrow 0 .
$$

Since $M$ is torsion free and divisible

$$
\operatorname{Ext}^{1}\left(H_{n-1}(G, Z), M\right)=\operatorname{Hom}\left(H_{n}(G, Z), M\right)=0 .
$$

Hence $H^{n}(G, M)=0$ and similarly $H^{n}(H, M)=0$ and the assertion follows. Henceforth we assume that the theorem has been proved for finitely generated nilpotent group $K$ with $k(K)<k(G)$.

In a nilpotent group $G$ the set $A$ of torsion elements form a normal subgroup [3, Vol. II, p. 215]. Thus the quotient group $G / A$ is torsion free and contains $H A / A$ which is isomorphic to $H / H \cap A$. The group $A$ is finite because by refining the series $G \triangleright A \triangleright\{e\}$ in to a chief series

$$
G \triangleright G_{1} \triangleright \cdots \triangleright G_{m}=A \triangleright A_{1} \triangleright \cdots \triangleright A_{n}=\{e\}
$$

the factor groups $A_{i} / A_{i+1}$ are necessarily finite cyclic groups. Hence $H^{n}(A, M)=0$ for all $n>0$. It follows from the Hochschild and Serre spectral sequence

$$
E_{2}^{m, n}=H^{m}\left(G / A, H^{n}(A, M)\right) \Rightarrow H^{m+n}(G, M)
$$

that $H^{m}(G / A, M)$ is isomorphic to $H^{m}(G, M)$ via the inflation homomorphism. Since $H \cap A$ is the normal subgroup of the torsion elements of $H$, we also have that

$$
H^{n}(H, M) \cong H^{n}(H / H \cap A, M)=H^{n}(H A / A, M) .
$$

Replacing $G$ by $G / A$ if necessary, we may assume that $G$ is torsion free from now on. 
Let $F$ be an infinite cyclic group contained in the intersection of the normal subgroup $H$ and the center of the nilpotent group $G$. It is well known that $H^{n}(F, M)=0$ if $n>1$. Hochschild and Serre proved in [2] that

$$
\begin{gathered}
H^{m}(G / F, M) \underset{i}{\underset{i}{\rightarrow}} H^{m}(G, M) \underset{r^{\prime}}{\rightarrow} H^{m-1}\left(G / F, H^{1}(F, M)\right) \\
\underset{d_{2}^{\prime}}{\rightarrow} H^{m+1}(G / F, M) \underset{i}{\rightarrow} H^{m+1}(G, M)
\end{gathered}
$$

is an exact sequence and $d_{2}^{\prime}(u)=-c \cdot u$ where $c$ is the group extension

$$
1 \rightarrow F \rightarrow G \rightarrow G / F \rightarrow 1
$$

and $i$ is the inflation map, while $r^{\prime}$ is induced by restricting the first argument of a suitably selected cocycle, representing the cohomology class, to $H$. Identifying $H^{1}(F, M)$ with $M$, we have the following commutative diagram

$$
\begin{aligned}
H^{m-2}(G / F, M) & \rightarrow H^{m}(G / F, M) \rightarrow H^{m}(G, M) \\
f_{1} \downarrow & f_{2} \downarrow \quad f_{3} \downarrow \\
H^{m-2}(H / F, M) & \rightarrow H^{m}(H / F, M) \rightarrow H^{m}(H, M) \\
& \rightarrow H^{m-1}(G / F, M) \rightarrow H^{m+1}(G / F, M) \\
& \rightarrow H^{m-1}(H / F, M) \rightarrow H^{m+1}(H / F, M)
\end{aligned}
$$

where $f_{1}, f_{2}, f_{3}, f_{4}$ and $f_{5}$ are restriction maps. Since

$$
k(G / F)=k(G)-k(F)=k(G)-1<k(G)
$$

the induction hypothesis yields that $f_{1}, f_{2}, f_{4}$ and $f_{5}$ are isomorphisms. Hence by [5, lemma ], $f_{3}$ is an isomorphism. If $M$ is a ring and $a$ and $b$ belong to $H^{*}(G, M)$, then

$$
\operatorname{res}(a \cdot b)=\operatorname{res} a \cdot \operatorname{res} b .
$$

So the restriction map is a ring isomorphism between $H^{*}(G, M)$ and $H^{*}(H, M)$. This completes the proof.

REMARK. The integral cohomology groups of a finitely generated nilpotent group do not have the property indicated in Theorem 1 for the rational cohomology groups. Consider the finitely generated nilpotent group

$$
G=\left\{\left|\begin{array}{lll}
1 & x & y \\
0 & 1 & z \\
0 & 0 & 1
\end{array}\right| \mid x, y, z \in Z\right\}
$$


It contains a subgroup

$$
H=\left\{\left(\begin{array}{lll}
1 & 2 x & 2 y \\
0 & 1 & 2 z \\
0 & 0 & 1
\end{array}\right) \mid x, y, z \in Z\right\}
$$

of index 8. Using spectral sequence, we obtain that $H^{2}(G, Z)=Z \oplus Z$, whereas $H^{2}(H, Z)=Z_{2} \oplus Z \oplus Z$. It can be easily derived from tha Universal Coefficient Theorem that in general the rational cohomology ring of a finitely generated nilpotent group $G$ equals $H^{*}(G, Z) \otimes Q^{+}$. Hence under the assumption of Theorem $1, H^{n}(G, Z)$ and $H^{n}(H, Z)$ merely have the same number of infinite cyclic direct summands.

One might think that perhaps solvability of the group $G$ would be sufficient to guarantee that $H^{n}\left(G, Q^{+}\right) \cong H^{n}\left(H, Q^{+}\right)$. Unfortunately, the torsion free, solvable group $G$ generated by $a$ and $b$ with defining relation $a b a^{-1}=b^{-1}$ has $H^{1}\left(G, Q^{+}\right)$isomorphic to $Q^{+}$, while its subgroup $H$ of index 2 generated by $a^{2}$ and $b$ has $H^{1}\left(H, Q^{+}\right)$isomorphic to $Q^{+} \oplus Q^{+}$.

For a nilpotent group, finite generation is equivalent to the maximum condition. We would thus like to investigate the situation when the maximum condition is replaced by the minimum condition. Our result is as follows.

THEOREM 2. If $G$ is a solvable group with minimum condition, then $H^{n}\left(G, Q^{+}\right)=0$ for $n>0$.

It follows from this theorem that under the above assumption $H^{n}\left(G, Q^{+}\right)=H^{n}\left(H, Q^{+}\right)$for any subgroup $H$ of $G$, because subgroups of $G$ are solvable and have minimum condition.

By Cernikov's Theorem [3, Vol. II, p. 191], we have the exact sequence

$$
1 \rightarrow H \rightarrow G \rightarrow K \rightarrow 1
$$

where $H$ is isomorphic to a finite direct sum of groups of type $Z_{p^{\infty}}$, the $p$-primary component of the group $Q^{+} / Z$, and $K$ is a finite solvable group. $H$ is therefore a locally finite group. Before proving Theorem 2 , we shall show the following theorem, which appears to have some interest of its own.

THEOREM 3. If $H$ is a locally finite group and $F$ is a field on which $H$ acts trivially, then

$$
H^{n}(H, F)=\text { proj } \lim H^{n}\left(H_{\alpha}, F\right)
$$

where $H_{\alpha}$ ranges over all the finitely generated subgroups of $G$. 
Proof. Let $Z(A)$ be the integral group ring of a given group $A$, and $B(A)$ denote the bar resolution of $A$ and $\bar{B}(A)_{n}$ the free abelian group generated by the $Z(A)$-free generators of $B(A)_{n}$. It is then immediate that inj $\lim Z\left(H_{\alpha}\right)=Z(H)$ and inj $\lim \bar{B}\left(H_{\alpha}\right)_{n}=\bar{B}(H)_{n}$ where $H_{\alpha}$ ranges over all finitely generated subgroups of $H$. Since $H$ is locally finite, $H_{\alpha}$ is necessarily finite. Hence $B\left(H_{\alpha}\right)_{n}$ is a finitely generated free abelian group and

$$
\operatorname{Hom}_{Z\left(H_{\boldsymbol{\alpha}}\right)}\left(B\left(H_{\alpha}\right)_{n}, F\right)=\operatorname{Hom}_{Z}\left(\bar{B}\left(H_{\alpha}\right)_{n}, F\right)
$$

is a finite dimensional vector space over $F$. Hence

$$
\begin{aligned}
\operatorname{Hom}_{Z(H)}\left(B(H)_{n}, F\right) & =\operatorname{Hom}_{Z(H)}\left(\bar{B}(H)_{n} \otimes_{Z} Z(H), F\right) \\
& =\operatorname{Hom}_{Z}\left(\bar{B}(H)_{n}, F\right) \\
& =\operatorname{Hom}_{Z}\left(\operatorname{inj} \lim \bar{B}\left(H_{\alpha}\right)_{n}, F\right) \\
& =\operatorname{proj} \lim \operatorname{Hom}_{Z}\left(\bar{B}\left(H_{\alpha}\right)_{n}, F\right) \\
& =\operatorname{proj} \lim \operatorname{Hom}_{Z\left(H_{\alpha}\right)}\left(B\left(H_{\alpha}\right)_{n}, F\right) .
\end{aligned}
$$

Let $D(A, n)$ and $Z(A, n)$ be the subgroup of coboundaries and the subgroup of cocycles in $\operatorname{Hom}_{Z(A)}\left(B(A)_{n}, F\right)$ respectively. Then we have the following exact sequence of finite dimensional vector spaces:

$$
\begin{aligned}
0 & \rightarrow Z\left(H_{\alpha}, n\right) \rightarrow \operatorname{Hom}_{Z_{\left(H_{\alpha}\right)}}\left(B\left(H_{\alpha}\right)_{n}, F\right) \stackrel{\delta}{\rightarrow} \operatorname{Hom}_{Z_{\left(H_{\alpha}\right)}}\left(B\left(H_{\alpha}\right)_{n}, F\right) \\
& \rightarrow D\left(H_{\alpha}, n+1\right) \rightarrow 0 .
\end{aligned}
$$

Inverse limit preserves the exactness of a sequence of finite dimensional vector spaces, so we get the exact sequence

$$
\begin{aligned}
0 \rightarrow \operatorname{proj} & \lim Z\left(H_{\alpha}, n\right) \rightarrow \operatorname{Hom}_{Z(H)}\left(B(H)_{n}, F\right) \\
& \stackrel{\delta}{\rightarrow} \operatorname{Hom}_{(H)}\left(B(H)_{n+1}, F\right) \rightarrow \operatorname{proj} \lim D\left(H_{\alpha}, n+1\right) \rightarrow 0 .
\end{aligned}
$$

It follows that

$$
\begin{gathered}
\text { proj } \lim Z\left(H_{\alpha}, n\right)=Z(H, n) \quad \text { and } \\
\text { proj } \lim D\left(H_{\alpha}, n+1\right)=D(H, n+1) .
\end{gathered}
$$

Taking the inverse limit of each term in the exact sequence of finite dimensional vector spaces

$$
0 \rightarrow D\left(H_{\alpha}, n\right) \rightarrow Z\left(H_{\alpha}, n\right) \rightarrow H^{n}\left(H_{\alpha}, F\right) \rightarrow 0
$$

we see that

$$
0 \rightarrow D(H, n) \rightarrow Z(H, n) \rightarrow \text { proj } \lim H^{n}\left(H_{\alpha}, F\right) \rightarrow 0
$$


is exact, and the theorem follows.

Corollary 1. $H^{n}\left(Z_{p^{\infty}}, Q^{+}\right)=0$ if $n>0$.

Proof. Every finitely generated subgroup of $Z_{p^{\infty}}$ is a cyclic group of order a power $p^{s}$ of $p$. Since $H^{n}\left(Z_{p^{s}}, Q^{+}\right)=0$ for $n>0$, we conclude that

$$
H^{n}\left(Z_{p^{\infty}}, Q^{+}\right)=\operatorname{proj} \lim H^{n}\left(Z_{p^{s}}, Q^{+}\right)=0 .
$$

We now turn to the proof of Theorem 2. Since the group $H$ is isomorphic to a finite direct sum of groups of type $Z_{p^{\infty}}$, its finitely generated subgroups $H_{\alpha}$ are finite. Hence $H^{n}\left(H_{\alpha}, Q^{+}\right)=0$ if $n>0$ and

$$
H^{n}\left(H, Q^{+}\right)=\text {proj } \lim H^{n}\left(H_{\alpha}, Q^{+}\right)=0 .
$$

The spectral sequence

$$
E_{2}^{m, n}=H^{m}\left(G / H, H^{n}\left(H, Q^{+}\right)\right) \Rightarrow H^{m+n}\left(G, Q^{+}\right)
$$

has $E_{2}^{m, n}=0$ for $n>0$. It follows that

$$
H^{m}\left(G, Q^{+}\right)=H^{m}\left(G / H, Q^{+}\right)=H^{m}\left(K, Q^{+}\right)=0
$$

as $K$ is a finite group. This completes the proof.

\section{REFERENCES}

1. K. Hirsch, On infinite solvable groups. I, Proc. London Math. Soc. (2) 44 (1938), 53-60.

2. G. Hochschild and J. Serre, Cohomology of group extensions, Trans. Amer. Math. Soc. 74 (1953), 110-134.

3. K. A. Kurosh, Theory of groups, Vols. I and II, Chelsea, New York, 1960.

4. A. I Mal'cev, Nilpotent torsion free groups, Izv. Akad. Nauk SSSR Ser. Mat. 13 (1949), 201-212. (Russian)

5. K. Nomizu, On the cohomology of compact homogeneous spaces of nilpotent Lie groups, Ann. of Math. 59 (1954), 531-538.

The University of Chicago 